\title{
Direction-Dependent Properties in Inverted Carbon Nitride Colloidal Glasses with Gradient Porosity
}

Jochen Bahner $^{[b]}$, Lukas Dobler ${ }^{[b]}$, Marvin Frisch ${ }^{[b, c]}$, Lars Vogelsang ${ }^{[b]}$, Helmut Cölfen ${ }^{[b]}$ and Sebastian Polarz ${ }^{[a, b] *}$

[a] Prof. Dr. S. Polarz

Leibniz-University Hannover, Institute of Inorganic Chemistry, Callinstrasse 9, 30167

Hannover, Germany

E-mail: sebastian.polarz@aca.uni-hannover.de

[b] J. Bahner, L. Dobler, M. Frisch, L. Vogelsang, Prof. Dr. H. Cölfen, Prof. Dr. S. Polarz University Konstanz, Department of Chemistry, Universitätsstrasse 10, 78457 Konstanz, Germany

[c] M. Frisch

Technical University of Berlin, Department of Technical Chemistry, Strasse des 17. Juni 124, 10623 Berlin, Germany

Keywords: Functional Gradient Materials; Porous Materials; Carbon Nitride; Inverted Opals; Analytical Ultracentrifugation

Abstract: It is well known that the step from a dense packing of colloidal beads to the inverted systems was important for the optimization of photonic crystal properties. Inverted opals made of high-refractive index semiconductors have attracted great attention due to their supreme optical features such as the occurrence of a photonic band-gap and because of an astonishing behavior in photocatalysis or for photovoltaics caused by so-called slow photons. It is much less known that photonic glasses, despite being disordered, exhibit unique optical properties too like random lasing or high-contrast structural colors. In analogy to opals and inverted opals, one can expect that inverted colloidal glasses may lead to an amplification of photonic properties as well or even to the emergence of unexpected features. An inverted photonic glass is characterized by a dense packing of monodisperse voids with colloidal dimensions without any long-range order. The preparation of inverse photonic glasses has rarely been reported by now and cases for materials composed of a semiconductor as a pore-wall material are unknown. The synthesis of porous carbon nitride $\left(\mathrm{C}_{3} \mathrm{~N}_{4}\right)$ with inverted colloidal glass structure is demonstrated here using a template approach. The formation of the template with glass-like order is achieved by analytical ultracentrifugation (AUZ) of size-selected silica colloids, 
followed by infiltration of a precursor sol, transformation to carbon nitride and the final removal of the template. The use of AUZ is particularly important because it even allows to use a mixture of differently sized template particles, which are gradually fractionated. Monoliths with optimized morphological features exhibiting a gradient porosity and highly accessible pores are obtained. The result are materials with a graded structure. What makes such functional gradient material interesting is, a dependence of the optical features on the position can be expected. In addition, the method presented here allows to synthesize materials with adjustable composition ranging from carbon over nitrogen-doped carbon to $\mathrm{C}_{3} \mathrm{~N}_{4}$ with either graphitic or polymeric structure. Therefore, the optical band gap is highly adjustable and tunable with regards to the photonic properties, as confirmed by optical absorption and photoluminescence measurements.

\section{Introduction}

Emerging energy technologies ask for a precise control over the functionality and morphology of porous solids. ${ }^{[1]}$ Within this class of compounds, the tailored combination of various pore sizes with different network-building materials has led to improved functional properties for a plethora of applications like catalysis and separation. ${ }^{[2]}$ The optimization of porosity for sustainability and better performance in the field of energy conversion and storage are among the most promising fields of research in material chemistry. ${ }^{[1 b, 3]}$ The synthesis of porous materials is mainly realized using so-called nanocasting processes employing colloidal or selfassembled templates. ${ }^{[4]}$ As most templating nanoparticles form colloidal crystals during assembly, dielectric structures, called photonic crystals, with a photonic band structure are formed. ${ }^{[5]}$ This property is maintained respectively even improved if the structures are inverted into a porous material, as it is a result of the periodically changing refractive index of the materials dictated by their structure. ${ }^{[6]}$ The synthesis of these so called inverse opals can be realized by infiltrating preformed colloidal templates with network forming precursors resulting 
in promising applications, for example as sensors, in photovoltaics or in batteries. ${ }^{[7]}$ Especially the synthesis of semiconductors, mainly titanium dioxide $\left(\mathrm{TiO}_{2}\right)$, has drawn intensive interest in research, as the photonic band structure can enhance the photocatalytic activity. ${ }^{[8]}$ Besides the advantageous high accessible surface of porous materials in general, the periodically changing refractive index between the network forming $\mathrm{TiO}_{2}$ and air filled voids leads to enhanced properties in photocatalysis. ${ }^{[9]}$ This leads to a localization of the light in the high refractive index $\mathrm{TiO}_{2}{ }^{[10]}$, enhanced light harvesting by light trapping ${ }^{[11]}$ as well as the slow photon effect ${ }^{[12]}$. An impressive study was reported by Low et al. using layered $\mathrm{TiO}_{2}$ inverse opals with varying pore sizes for the photocatalytic $\mathrm{CO}_{2}$ reduction. The combination of two different photonic structures resulted in a significantly enhanced catalytic activity due to improved light harvesting. ${ }^{[13]}$

In contrast to highly ordered photonic crystals, the experimental realization of amorphous structured photonic glasses has been rarely reported. ${ }^{[14]}$ While special photonic properties like random lasing ${ }^{[15]}$, isotropic structural color ${ }^{[16]}$ and Anderson localization of light ${ }^{[17]}$ have been described for colloidal photonic glasses ${ }^{[18]}$, few investigations on the inverted porous structures have been performed. While some process has been made towards inverted photonic glasses consisting of ceramics ${ }^{[19]}$ or polymers ${ }^{[20]}$, the investigation of the properties is focused towards structural colour and therefore isolating materials. ${ }^{[21]}$ Because of the correlation between the photonic band structure as a function of the opal structure and the electronic gap and related properties of the semiconductor material, it could be highly interesting to obtain materials with pore features as a function of position.

However, the vast majority of research in porous materials in general and in inverted opals in particular focuses on a statistical distribution of all structural and functional features, resulting in homogeneity. If properties of a material are not separated by a sharp boundary, but gradually change into a spatial direction, so called functional gradient materials (FGMs) are formed. ${ }^{[22]}$ The field of FGMs is quite well researched for materials exhibiting functional chemical 
gradients $^{[23]}$ with promising applications for instance in the synthesis of tailored adhesive coatings ${ }^{[24]}$ or in separation science ${ }^{[25]}$. Materials characterized by a gradually changing porosity are common in nature, e.g. in bones ${ }^{[26]}$, leading to their high mechanical stability, low density and highly accessible pores. ${ }^{[27]}$ The synthesis of such materials has been reported for large pores $(>10 \mu \mathrm{m})$ with applications e.g. in tissue engineering ${ }^{[28]}$ or electrophoresis ${ }^{[29]}$. Applied methods include 3D printing ${ }^{[30]}$, emulsion templating ${ }^{[31]}$ or freeze-casting ${ }^{[32]}$. Stubenrauch and coworkers reported another innovative pathway. ${ }^{[33]}$ Using a self-build combination of microfluidics and an extrusion printer, polymer hydrogels and their composites are synthesized with gradually changing pore sizes between 80 and $800 \mu \mathrm{m}$. For gradients including smaller pores in the nanometer regime, a selective swelling of copolymers can be employed. ${ }^{[34]}$ However, the experimental realization of well-defined gradients in macroscopic materials is challenging. The main limitation is the demanding generation of suitable templates, as well as their infiltration. A promising pathway to overcome this problem is the application of gradient centrifugation, which has seen a rise in research towards the structuration of colloidal assembly. ${ }^{[35]}$ While significant advances have been made on the understanding of the assembly behavior of monodisperse spheres under centrifugal force, ${ }^{[36]}$ the occurrence of non-dense structures with large scale gradients in particle sizes has scarcely been reported ${ }^{[35 b]}$ Our group has reported the possibility of creating various ordered as well as gradually changing structures when ultracentrifugation was used to assemble binary mixtures of polystyrene nanoparticles. ${ }^{[37]}$ This behavior could be adapted towards a templating process, resulting in the first synthesis of a gradient porous carbon material with pores on the nanoscale, which showed direction dependent enhanced functional properties when applied as electrode in lithium-oxygen batteries. ${ }^{[38]}$ Based on the latter publication and the arguments given for graded inverted opals, we ask if the method can be used for the generation of materials composed of a semiconductor as well. 
Carbon nitride $\left(\mathrm{C}_{3} \mathrm{~N}_{4}\right)$ is a semiconductor, which is obviously strongly related to carbon materials. It is known, that carbon materials can be tuned by the incorporation of heteroatoms into the carbon network. ${ }^{[39]}$ The most discussed dopant is nitrogen due to the fact that the control over the degree of incorporation into the carbon network allows the adjustment of the functional properties for various applications, such as catalytic activity in electrochemistry or adsorption capacity for $\mathrm{CO}_{2}$-capture. ${ }^{[40]}$ Low to medium doping levels of nitrogen into carbon can lead to highly active electrocatalysts applicable towards the oxygen reduction reaction in lithium-based batteries $^{[41]}$ or the electro-reduction of carbon dioxide ${ }^{[42]}$. At very high levels of nitrogen incorporated into the carbon network, $\mathrm{C}_{3} \mathrm{~N}_{4}$ is formed. The family of $\mathrm{C}_{3} \mathrm{~N}_{4}$ derived compounds is characterized by a visible band gap which can be adjusted in the range of $\sim 1.4-2.8 \mathrm{eV}$ depending on the stoichiometric composition ${ }^{[43]}$ and crystalline structure ${ }^{[44]} \cdot \mathrm{C}_{3} \mathrm{~N}_{4}$ attracts additional significant interest in research as it is eco-friendly, cheap and stable. ${ }^{[45]}$ Additionally, the energy levels of valence and conduction bands are suitable for hydrogen $\left(\mathrm{H}_{2}\right)$ generation and for Z-scheme based photocatalytic water purification. Therefore, applications in these fields are currently rising. ${ }^{[46]}$ Especially the control over the pore sizes and structures leads to enhanced photocatalytic properties, in example for water splitting or $\mathrm{CO}_{2}$ reduction. ${ }^{\text {[6d, }}{ }^{47]}$ Several researchers have already made notable progress on porous $\mathrm{C}_{3} \mathrm{~N}_{4}$ materials. For instance, Liang et al. used monolithic porous $\mathrm{C}_{3} \mathrm{~N}_{4}$ as catalyst for photocatalytic water splitting. ${ }^{[48]}$ The enhanced turnover rate of the macroscopic structure was attributed to a better light capture, charge separation and mass diffusion, which could be further improved in monoliths with an ordered porosity as reported by Sun and coworkers. ${ }^{[49]}$

In a recent paper ${ }^{[50]}$, we showed that nitrogen and other dopants can be introduced into the pure carbon materials described before ${ }^{[38]}$. The nitrogen atoms were introduced by the template particles, e.g. poly-aniline modified polystyrene lattices. However, our results showed clearly $^{[50]}$, that the maximum amount of incorporated nitrogen is insufficient for the conversion to $\mathrm{C}_{3} \mathrm{~N}_{4}$. Therefore, we refrain from using template particles as nitrogen source, and present a 
newly developed approach. Accordingly, inverted colloidal glasses consisting of the semiconductor $\mathrm{C}_{3} \mathrm{~N}_{4}$ with a gradient structure in porosity can now be afforded. The paper is organized as follows. First, we will show how colloidal photonic glasses, consisting of differently sized silica nanoparticles ( $\left.\mathrm{SiO}_{2}-\mathrm{NPs}\right)$, can be formed using preparative ultracentrifugation (PUC). We will then present the applicability of these novel structures as templates for the generation of gradient porous materials. The infiltration of the structures with commercially available precursors will ultimately result in the first synthesis of gradient porous graphitic $\left(\mathrm{g}-\mathrm{C}_{3} \mathrm{~N}_{4}\right)$ and polymeric $\left(\mathrm{p}-\mathrm{C}_{3} \mathrm{~N}_{4}\right)$ carbon nitrides. Finally, the optical properties of the materials are studied.

\section{Results and Discussion}

As described before, we could successfully use binary mixtures of colloidal dispersions of two monodisperse polystyrene spheres (PS) for the co-assembly with a resorcinol-aldehyde (RA) precursor mixture using ultracentrifugation. ${ }^{[38,50-51]}$ The desired carbon materials were obtained after crosslinking to a RA-gel followed by carbonization accompanied by thermal removal of PS. Our initial idea was to substitute the RA process by reactions, which are known from literature to end up in different carbon nitride compounds. ${ }^{[44 b, 52]}$ Although, the conversion to carbon nitride materials was successful as shown for a guanidine-based approach in Supporting Information Figure S1, the structural information is lost entirely. Instead of an inverted opal structure, a porous agglomerate of ill-defined particles in the size-range $100 \mathrm{~nm}-1 \mu \mathrm{m}$ was obtained according to scanning electron microscopy (SEM). Extensive work showed that PS latexes are unsuitable as templates. Therefore, we developed a more flexible approach as shown in Scheme 1. Using $\mathrm{SiO}_{2}$-nanoparticles ( $\left.\mathrm{SiO}_{2}-\mathrm{NPs}\right)$ results in the flexibility to tune the precursor composition (Supporting Information Scheme S1) towards the successful synthesis of 
monolithic materials with compositions ranging from carbon over nitrogen doped carbon towards carbon nitride.

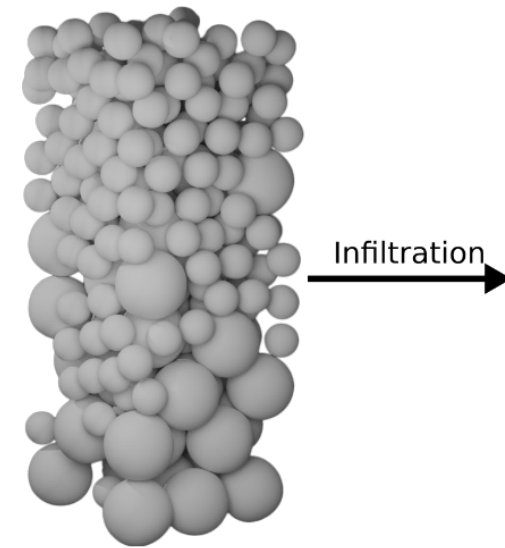

Photonic Glass Gradient Template

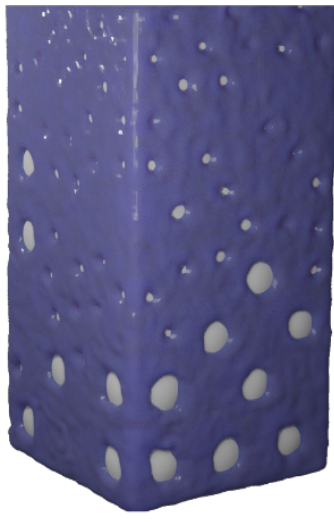

Silca/Precursor Composite

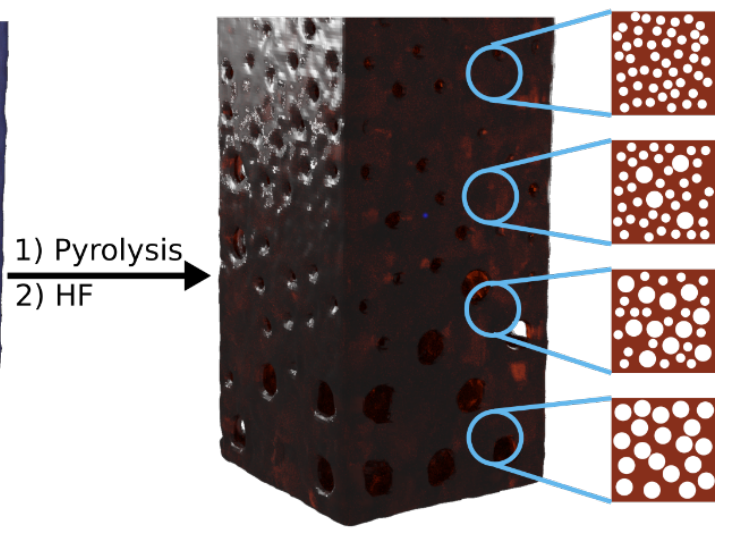

Gradient Porous Monolith with Adjustable Composition

Scheme 1. Schematic illustration of the synthetic approach towards gradient porous monoliths with adjustable nitrogen contents. First, gradient templates are formed by ultracentrifugation. These can be infiltrated either in situ or by melt infiltration with different precursors as shown in Scheme S1. After a thermal conversion and template removal with hydrofluoric acid, gradient porous monoliths with adjustable compositions are yielded.

\subsection{Ultracentrifugation-assisted construction of gradient templates composed of silica spheres.}

Monodisperse spheres are also available for silica from the so-called Stöber method. ${ }^{[53]}$ The usage of colloidal glasses formed by $\mathrm{SiO}_{2}$-NPs as templates offers several advantages. First, $\mathrm{SiO}_{2}$-NPs can form stable dispersions in a wide range of precursor solutions. Second, $\mathrm{SiO}_{2}-\mathrm{NPs}$ sediment in high density solutions, allowing for a precise control over the assembled structure. This was not possible using PS-NPs, which float on dense solvents when the solvent density exceeds $1.05 \mathrm{~g} / \mathrm{mL}$. Thus, we show results for the assembly behavior of binary mixtures of $\mathrm{SiO}_{2}$-NPs inside centrifugal fields, resulting in the formation of gradient templates. We aim to create a slight separation between the particles, resulting in a gradual change in the local concentrations of the differently sized particles. Investigations of the assembly behavior of monodisperse colloids have drawn renewed interest in research ${ }^{[35 b]}$ as colloidal assembly is a promising pathways towards the creation of nanoparticle-based materials. ${ }^{[54]}$ However, most 
research is focused on the assembly into homogenous and highly ordered structures. The reason for this is, that contrary to intuition, the formation of highly ordered structures from monodisperse nanoparticles is much more favorable than our desired disordered gradient structure. ${ }^{[14,18,37]}$ We have prepared colloidal silica spheres with diameters of $129 \pm 14 \mathrm{~nm}$ (denoted as $\left.\mathrm{SiO}_{2}-129\right)$ and $310 \pm 20 \mathrm{~nm}$ (denoted as $\left.\mathrm{SiO}_{2}-310\right)$; the characterization is given in Supporting Information Figure S2. The assembly behavior under centrifugal force was then analyzed in situ using analytical ultracentrifugation (AUC), because recent studies have shown that AUC is very suitable to reveal information about the sedimentation of binary mixtures of small $\mathrm{SiO}_{2}-\mathrm{NPs}^{\left[{ }^{[36]}\right]}$ It is worth noting that the pure $\mathrm{SiO}_{2}-129$ and $\mathrm{SiO}_{2}-310$ both show a typical sedimentation behavior expected for monodisperse samples. As shown in Supporting Information Figure S3, a constant decrease in nanoparticle concentration with increasing time of centrifugation can be observed. In contrast, the sedimentation behavior of binary mixtures of $\mathrm{SiO}_{2}-129$ and $\mathrm{SiO}_{2}-310$ presented in Figure 1a is characterized by the formation of a plateau in the local NP concentration separating the two steps representing each particle size. This is a clear indication of the temporal separation of the individual NPs sedimentation. 

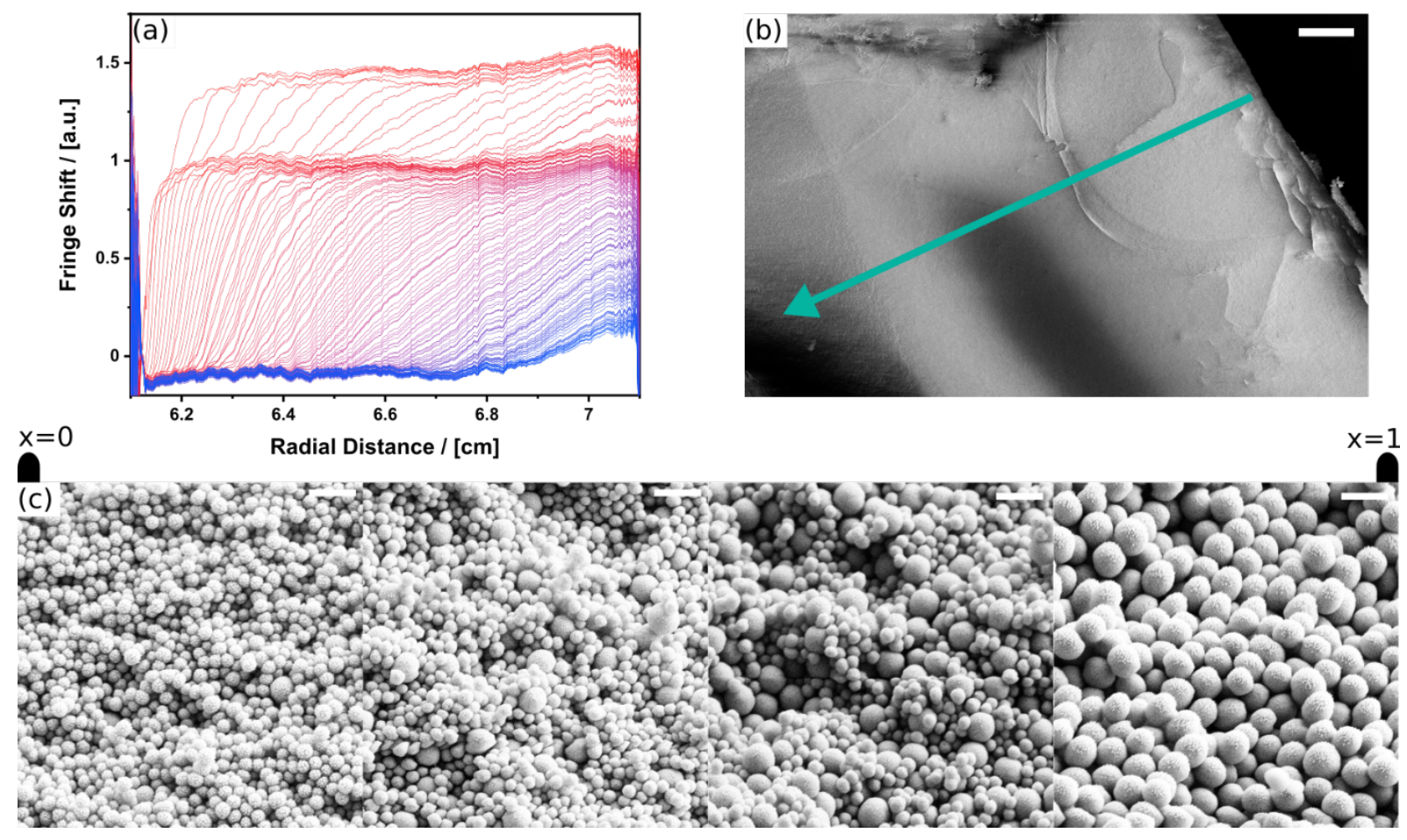

Figure 1. (a) Radial Rayleigh interference scans over time from red to blue in a sedimentation velocity run of a binary mixture of $\mathrm{SiO}_{2}-129$ and $\mathrm{SiO}_{2}-310$ at a rotational speed of $1500 \mathrm{rpm}$. The time interval between two scans is $120 \mathrm{~s}$. SEM micrographs of (b) a Cross-section of silica opal with gradient in particle size, arrow indicating the direction of the gradient; scalebar $=50 \mu \mathrm{m}$ and (c) micrographs taken along the gradient indicating the gradient in particle size; scalebar $=500 \mathrm{~nm}$. Micrographs at lower magnifications given in the Supporting Information Figure S4.

In binary mixtures, $\mathrm{SiO}_{2}-310$ completely and rapidly sediments within the first $30 \mathrm{~min}$ of centrifugation, as indicated by a decrease in maximal fringes above the plateau. A complete sedimentation of $\mathrm{SiO}_{2}-129$ can only be observed after 150 min. However, a decrease in concentration of $\mathrm{SiO}_{2}-129$, detectable by a slight loss in intensity at the plateau, is detected from the start of the sedimentation velocity experiment as an effect of radial dilution in the sectorshaped AUC centerpieces. In addition, sedimentation occurs only partially separated and a gradient formation seems possible. To further investigate this, we performed additional AUC measurements using UV-detection and compared them with simulations (see Supporting Information Figure S3b), confirming our findings of a gradual fractionation according to the particle size. Interactions between the different particle species can be excluded as the 
hydrodynamic diameters calculated from the presented AUC data (see Supporting Information Figure S3c) are identical to the values determined from monomodal samples. As AUC is limited to highly diluted samples and therefore hardy applicable to the synthesis of macroscopic materials, we tested the transfer of the observed gradual separation to concentrated solutions and larger scales using PUC. After sedimentation of binary mixtures of $\mathrm{SiO}_{2}-129$ and $\mathrm{SiO}_{2}-310$ at intermediate centrifugal forces ${ }^{[37]}$, a monolithic colloidal structure with macroscopic dimensions as shown in Figure 1b was formed after drying. Using SEM, we investigated the structuration of the nanoparticles at distinct points along the direction of the centrifugal force indicated by the arrow in the cross-section. As predicted by AUC measurements, mixtures of $\mathrm{SiO}_{2}-129$ and $\mathrm{SiO}_{2}-310$ assemble in a gradient structure shown in Figure 1c. While the upper part of the monolith $(\mathrm{x} \rightarrow 0)$ is composed of only $\mathrm{SiO}_{2}-129$, showing some degree of closerange order in an overall glassy state, the local fraction of $\mathrm{SiO}_{2}-310$ increases gradually along the direction of the centrifugal force. Finally, in the lower part of the gradient structure $(x \rightarrow 1)$ only large particles, again assembled in a glassy state, can be observed. For intermediate positions, no ordered aggregates are observed, indicating a kinetically driven assembly, overcoming the expected formation of ordered aggregates over the whole monolith (see also Supporting Information Figure S4). ${ }^{[14,55]}$ The assembly behavior can easily be adjusted by the relative ratio of the individual particles and centrifugal force. At higher centrifugal forces, ordered structures of $\mathrm{SiO}_{2}-129$ can be observed and no regions consisting of exclusively $\mathrm{SiO}_{2}-310$ can be observed as the initial sedimentations occurs rapidly (see Supporting Information Figure S5).

\subsection{Carbon nitride materials with gradient porosity.}

The disordered, but dense packings of silica spheres presented in the last paragraph can now be used as templates (Scheme 1). The viability of the approach can be proven by using the silica 
template instead of PS, but for the moment, for the preparation of a porous carbon material from a RA sol in analogy to ref. 38. During fractionation of the nanoparticles according to their size by PUC, the voids in the template are filled by the present precursor. After drying, the formed composite is converted to the final product by a single pyrolysis step. Gradient porous graphitic carbon materials were obtained successfully (see Supporting Information Figure S6). Beneficially, the detailed investigation of the infiltration and network forming process can be adopted towards the synthesis of a broader range of carbon-based materials with graded porosity, which was not possible using PS templates. Exemplarily, Figure S7 shows the successful synthesis of highly graphitized carbon materials with gradient porosity using $\mathrm{SiO}_{2}$-NPs dispersed in furfuryl alcohol. Lightly nitrogen doped carbons are accessible by infiltration with pyrrole acting as precursor and nitrogen doping agent simultaneously. However, the usage of disordered colloidal assemblies of $\mathrm{SiO}_{2}-\mathrm{NPs}$ as templates offer possibilities in material design inaccessible until now. Due to the higher colloidal stability and density of $\mathrm{SiO}_{2}$-NPs compared to PS, a temporal separation between the template formation step and the gelation of the precursor sol is feasible. Consequently, we can now use more demanding precursor sols, which is the key for the synthesis of materials with high nitrogen contents increasing from nitrogen-doped carbon (N@C) to $\mathrm{C}_{3} \mathrm{~N}_{4}$ as shown in Figure 2. To achieve this, we dispersed binary mixtures of $\mathrm{SiO}_{2}-129$ and $\mathrm{SiO}_{2}-310$ in concentrated precursor solutions (see (I),(II) in Supporting Information Scheme S1), and fractionated the $\mathrm{SiO}_{2}$-NPs by their size using PUC, followed by a thermal transformation step and removal of the $\mathrm{SiO}_{2}$ template by $\mathrm{HF}$ etching. Depending on the nitrogen content of the precursor, either highly nitrogen doped carbon materials, further denoted as $\mathrm{N} @ \mathrm{C}$ (Figure 2(a)) or gradient porous g- $\mathrm{C}_{3} \mathrm{~N}_{4}$ (Figure 2(b)) is yielded. A third possibility (III, Supporting Information Scheme S1) to synthesize gradient porous materials is melt infiltration. We covered the monolithic templates with 5-aminotetrazole (5AT). A slow heat up to $550{ }^{\circ} \mathrm{C}$ leads to an infiltration with molten $5 \mathrm{AT}$ 
which then polymerizes to form $\mathrm{p}-\mathrm{C}_{3} \mathrm{~N}_{4}$ (Figure 2(c)). After etching with HF, orange monolithic $\mathrm{p}-\mathrm{C}_{3} \mathrm{~N}_{4}$ is obtained.
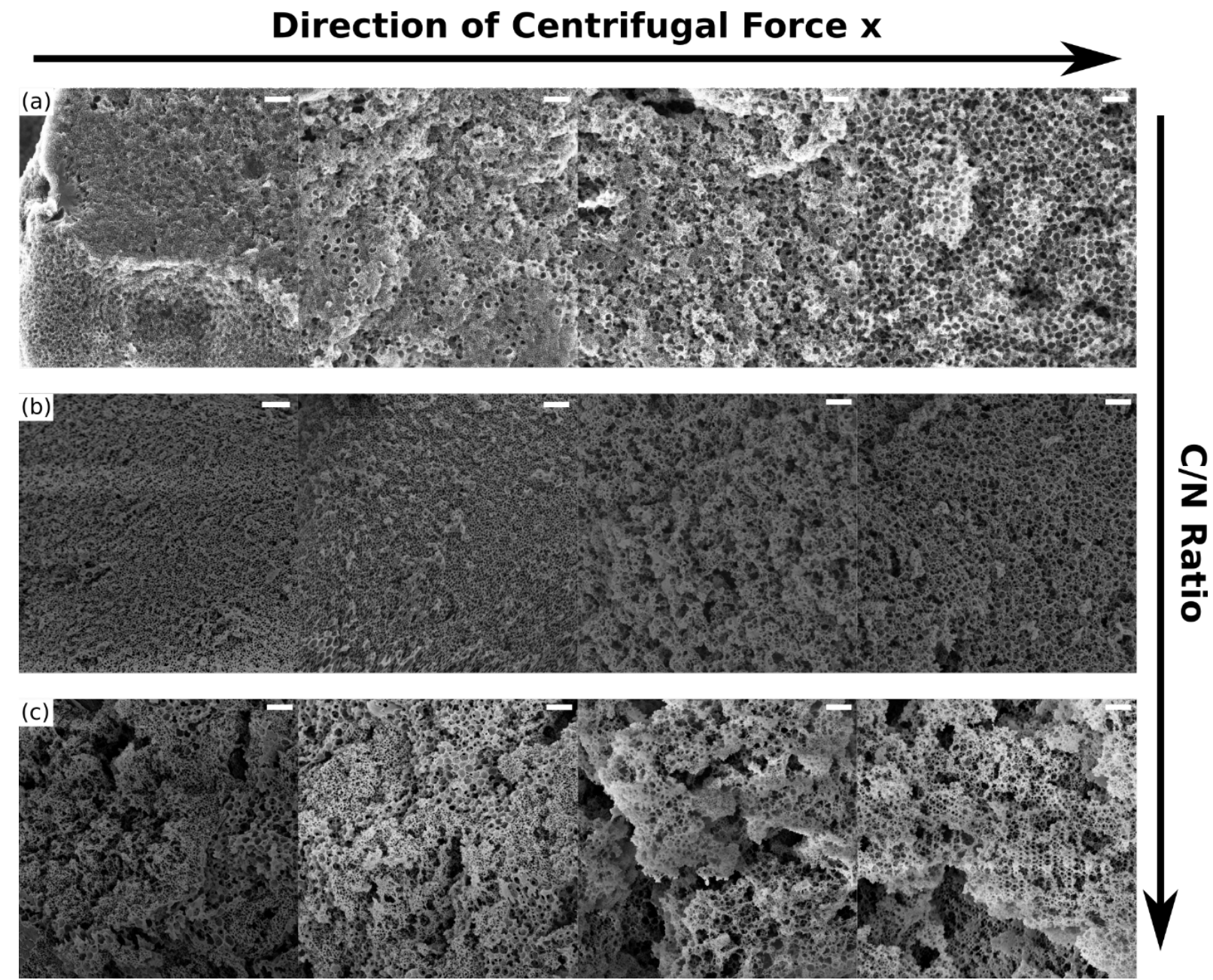

Figure 2. Gradient structure of inverted colloidal glasses with increasing nitrogen content from (a) to (c) as shown by subsequent SEM images taken along the internal coordinate $\mathrm{x}$ with scale bars of $1000 \mathrm{~nm}$. SEM micrographs for (a) highly nitrogen doped carbons $(\mathrm{C} @ \mathrm{~N})(\mathrm{b})$ graphitic carbon nitride $\left(\mathrm{g}-\mathrm{C}_{3} \mathrm{~N}_{4}\right)$ and (c) polymeric carbon nitride $\left(\mathrm{p}-\mathrm{C}_{3} \mathrm{~N}_{4}\right)$.

All materials were yielded in a monolithic cylindrical shape with dimensions of $\sim 0.7 \mathrm{~cm}$ and a gradient in pore sizes in the direction of the centrifugal force $(\sim 0.15 \mathrm{~cm})$. The presence of the gradient structure can be proven by SEM micrographs as shown in Figure 2. Evidently, only smaller pores with a diameter of $88 \pm 8 \mathrm{~nm}$ can be observed at the beginning of the gradient (left side, $x \rightarrow 0$ ). The portion of larger pores with average diameters of $316 \pm 27 \mathrm{~nm}$ gradually increases towards the end of the gradient (right side, $x \rightarrow 1$ ). The accessibility of the internal 
surface $\left(\sim 50 \mathrm{~m}^{2} / \mathrm{g}\right)$ completely contributed by macroporous can be confirmed by $\mathrm{N}_{2}$-physisorption analysis (see Supporting Information Figure S8). This is further confirmed by recording higher magnified SEM micrographs given in Figure S9 for all materials showing interconnected pores of uniform sizes. Importantly, the structural features do not significantly differ for the presented samples as evident by comparing the local pore sizes displayed for the individual samples presented in Figure 2(a)-(c) which is in accordance with the postulated formation process. To better compare the formed porosity gradients of the synthesized materials, we employed trainable Weka segmentation ${ }^{[56]}$ implemented in Image ${ }^{[57]}$, as previously reported $^{[50-51]}$. This allows an objective calculation of the local area fraction of small pores, templated from $\mathrm{SiO}_{2}-129$ at specific positions $\mathrm{x}$ in the gradient region. As shown in Figure 3, all presented materials exhibit a gradual changing pore size along the gradient of the centrifugal force, confirming the analysis from SEM micrographs. Due to the different synthetic procedures, the density of the dispersion changes, resulting in a slight change in sedimentation coefficients and therefore gradient structure. This is evident as $\mathrm{p}-\mathrm{C}_{3} \mathrm{~N}_{4}$ (red curve in Figure 3), synthesized by melt infiltration shows a sharp transition between regions with predominantly small and large pores as observed during the investigation of the assembly process. In contrast, the gradient region is extended for $\mathrm{C} @ \mathrm{~N}$ (black curve) and $\mathrm{g}-\mathrm{C}_{3} \mathrm{~N}_{4}$ (blue curve). This is a result of the high density of concentrated precursor solution, leading to a less pronounced fractionation, presenting an easy possibility to control the extension of the gradient region. 


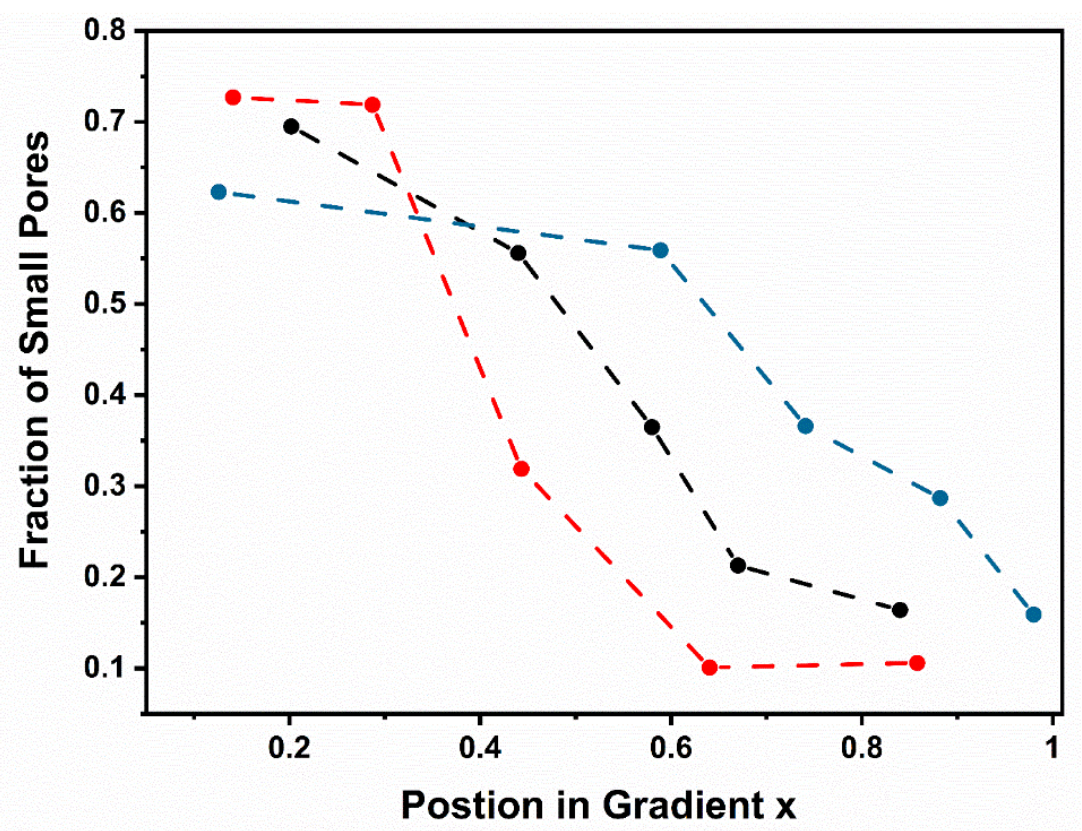

Figure 3: Correlation between the position in the gradient region and the calculated fraction of small pores according to WEKA image segmentation for $\mathrm{C} @ \mathrm{~N}$ (black), g- $\mathrm{C}_{3} \mathrm{~N}_{4}$ (blue) and $\mathrm{p}-\mathrm{C}_{3} \mathrm{~N}_{4}$ (red).

\subsection{Chemical structure of the carbon nitride materials.}

We know up to this point that monolithic glassy materials with the desired gradient porosity have been obtained by all presented methods, but it should be proven next, that the deliberately chosen methods lead to different kinds of materials. Therefore, a characterization of the chemical structure is necessary. First, we have investigated the network formation in the template's voids using thermogravimetric analysis (see Supporting Information Figure S10). For C@N (black curve), a stepwise mass loss is observed due to the elimination of water and ammonia during condensation reactions between $150{ }^{\circ} \mathrm{C}$ and $550{ }^{\circ} \mathrm{C}$. The final formation of a graphitic carbon networks occurs during an isothermal treatment at $550{ }^{\circ} \mathrm{C}$, resulting in the formation of a $\mathrm{C} @ \mathrm{~N}-\mathrm{SiO}_{2}$-composite. A similar slow and stepwise condensation under cleavage of ammonia and water is observed during the formation process of $g-\mathrm{C}_{3} \mathrm{~N}_{4}$ (blue curve) both synthesized after in situ infiltration. For $\mathrm{p}-\mathrm{C}_{3} \mathrm{~N}_{4}$ (red curve) synthesized by melt infiltration, a loss of water of crystallization from the precursor (5AT monohydrate) is detected at $\sim 100{ }^{\circ} \mathrm{C}$. Subsequently, the self-condensation of 5AT starts shortly after melting and infiltration at 
$\sim 200{ }^{\circ} \mathrm{C}$. After a rapid initial mass loss, the final $\mathrm{p}-\mathrm{C}_{3} \mathrm{~N}_{4} / \mathrm{SiO}_{2}$ composite is formed after slower secondary condensation reactions and after an isothermal treatment at $550{ }^{\circ} \mathrm{C}$. To clarify the underlying chemical processes leading to the network formation, we used ATR-IR given in Figure 4, and compared the spectra before and after pyrolysis. Besides the Si-O-Si-signal at $1051 \mathrm{~cm}^{-1}$, strongly visible in all samples due to the presence of $\mathrm{SiO}_{2}-\mathrm{NPs}$, important insight into the process can be gained by an evaluation of the additional signals assignable to the precursor present in voids. In case of $\mathrm{C} @ \mathrm{~N}$ (grey curve) strong signals are observed at $1668 \mathrm{~cm}^{-1}$ corresponding to $\mathrm{C}=\mathrm{O}$ vibrations and $1554 \mathrm{~cm}^{-1}$ assignable to ring deformations in triazine units stemming from melamine. As only minor amine signals in the range of $3200 \mathrm{~cm}^{-1}$ and $3600 \mathrm{~cm}^{-1}$ are observable, we conclude that the precursor sol consisting of formaldehyde, guanidine, and melamine undergoes condensation reactions during drying of the composite resulting in a fixation of the precursor network.

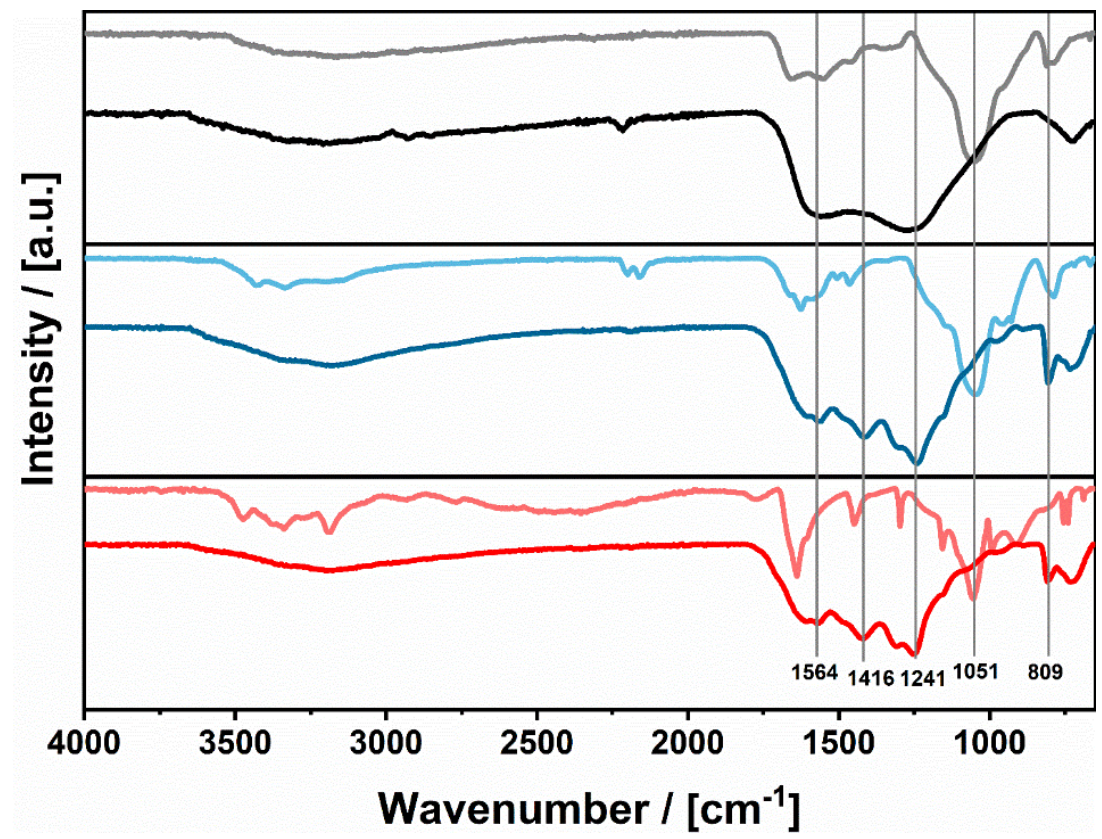

Figure 4: IR-spectra of the infiltrated precursor-SiO 2 -composites (light curves) and the final gradient porous materials after template removal by HF etching (dark curves) for $\mathrm{C} @ \mathrm{~N}$ (black), g- $\mathrm{C}_{3} \mathrm{~N}_{4}$ (blue) and $\mathrm{p}-\mathrm{C}_{3} \mathrm{~N}_{4}$ (red).

Similar observations can be made for the synthesis of $\mathrm{g}-\mathrm{C}_{3} \mathrm{~N}_{4}$ synthesized using mixtures of urea and cyanamide. The dried infiltrated template (light blue curve) shows a reduced intensity for amine groups between $3334 \mathrm{~cm}^{-1}$ and $3061 \mathrm{~cm}^{-1}$ in the infiltrated template after drying in 
comparison to untreated cyanamide (Supporting Information Figure S11). Additional C-N and $\mathrm{C}=\mathrm{N}$ bonds are observable between $1633 \mathrm{~cm}^{-1}$ and $1459 \mathrm{~cm}^{-1}$, indicating a self-condensation of cyanamide occuring during drying. The importance of these primary condensation reactions is shown by a comparison to the synthesis of $\mathrm{C}_{3} \mathrm{~N}_{4}$ using only guanidine, which remains unchanged during drying (Supporting Information Figure S12), resulting in a collapse of the network due to an insufficient stability. Therefore, we derived two requirements for the synthesis of monolithic gradient porous materials using in situ infiltration during template formation. First, a primary reaction of the precursor must occur during drying of the composite yielded after fractionation to fix the structure. Second, the final matrix-building material must densely fill the template's voids. Otherwise, voids and cracks persist in the final structure leading to a collapse of the monolithic structure and therefore to a formation of porous powders. As expected, the situation is different for the $\mathrm{p}-\mathrm{C}_{3} \mathrm{~N}_{4}$ prepared by melt infiltration, showing a physical mixture of 5AT and silica before pyrolysis (light red curve). After pyrolysis and template removal by HF, the signal corresponding to Si-O-Si disappears in all presented samples (dark curves in Figure 4). This indicates a complete removal of the template, which is confirmed by Energy-dispersive X-ray spectroscopy (EDX, Supporting Information Figure S13) showing no remaining silicon signal.

The binding situation between carbon and nitrogen atoms as well as the ratio of carbon to nitrogen in the class of carbon/nitrogen-based solids is important to control the optical properties of the resulting materials. ${ }^{[58]}$ The synthetic method presented here allows the precise control of the resulting functionalities. The IR spectrum of $\mathrm{N} @ \mathrm{C}$ (black curve) is characterized by broad, overlaying signals between $1660 \mathrm{~cm}^{-1}$ and $1050 \mathrm{~cm}^{-1}$ assignable to various $\mathrm{N}-\mathrm{C}$ and $\mathrm{N}=\mathrm{C}$ bonds in heterocycles and $\mathrm{C}=\mathrm{C}$ bonds stemming from the graphitic network. In contrast, g- $\mathrm{C}_{3} \mathrm{~N}_{4}$ (blue curve) and porous $\mathrm{p}-\mathrm{C}_{3} \mathrm{~N}_{4}$ (red curve) are characterized by separated strong signals in the range between $1650 \mathrm{~cm}^{-1}$ and $1250 \mathrm{~cm}^{-1}$. This is characteristic for a sp'-hybridized heterocycle with the signals at $1605 \mathrm{~cm}^{-1}$ and $1564 \mathrm{~cm}^{-1}$ corresponding to $\mathrm{C}=\mathrm{N}$ bonds and the 
signals at $1416 \mathrm{~cm}^{-1}, 1305 \mathrm{~cm}^{-1}$ and $1241 \mathrm{~cm}^{-1}$ assignable to $\mathrm{C}-\mathrm{N}$ bonds. Furthermore, the characteristic fingerprint signal of the triazine unit at $809 \mathrm{~cm}^{-1}$ is clearly visible, indicating the formation of stoichiometric $\mathrm{C}_{3} \mathrm{~N}_{4}{ }^{[46 c, 48]}$ To confirm the conclusions drawn from IR and gain information of the amount of nitrogen incorporation, we investigated the exact binding situation within the materials by X-ray photoelectron spectroscopy (XPS) given in Figure 5. Quantitative analysis reveals an atomic $\mathrm{C} / \mathrm{N}$ ratio of 1.61 for $\mathrm{C} @ \mathrm{~N}$ expected for nitrogen doped carbons synthesized at low carbonization temperatures. ${ }^{[59]}$ Accordingly, we expect the occurrence of distinguishable energy levels for nitrogen atoms as schematically shown in Figure 5a. The N 1s region (Figure 5b) shows contributions at $398.1 \mathrm{eV}$, which can be assigned to pyridinic nitrogen species (shown in magenta), and $399.6 \mathrm{eV}$ characteristic for pyrrolic nitrogen species shown in green respectively. ${ }^{[60]}$ In the C 1 s region (Figure 5c) two species can be observed as well. The signal at $284.8 \mathrm{eV}$ is characteristic of $\mathrm{C}=\mathrm{C}$ bonds while the second contribution at $287.2 \mathrm{eV}$ can be assigned to carbon atoms bound to nitrogen.

(a)

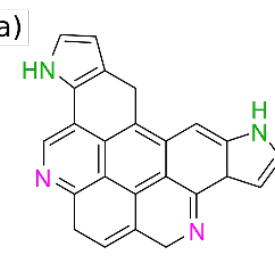

(f)

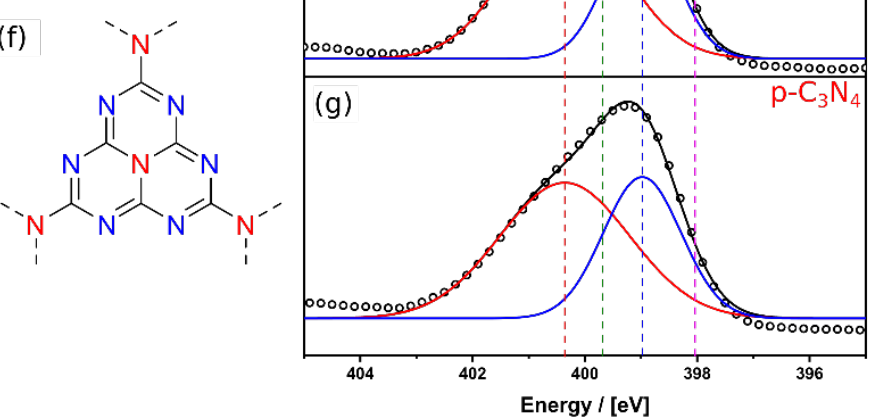

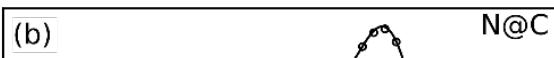

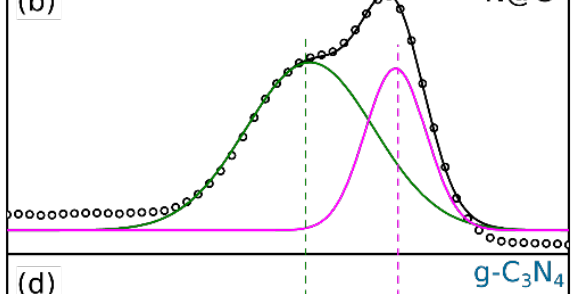

(d)

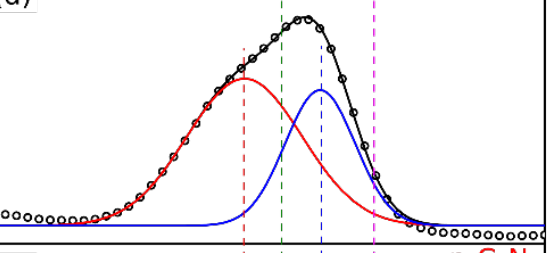

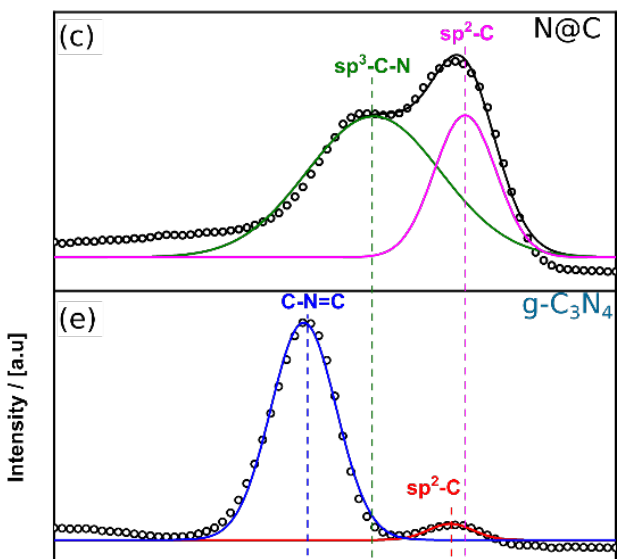

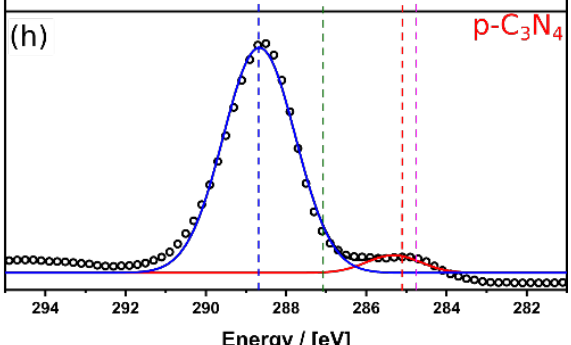

Figure 5: (a) Schematic depiction of the chemical structure of nitrogen doped carbon. Detailed XPS spectra for N@C of the (b) N 1s region and (c) C 1s region. Detailed XPS spectra for g-C3N4 of the (d) N 1s region and (e) C 1s region. (f) Schematic depiction of the chemical structure of carbon nitride. Detailed XPS spectra for g-C3N4 of the $(g) \mathrm{N} 1 \mathrm{~s}$ region and (h) $\mathrm{C}$ 1s region. 
This binding Situation in N@C is clearly distinguishable from the stoichiometric carbon nitride compound g- $\mathrm{C}_{3} \mathrm{~N}_{4}$ and $\mathrm{p}-\mathrm{C}_{3} \mathrm{~N}_{4}$. The corresponding $\mathrm{N}$ 1s spectra (Figure 5(d),(g)) are composed of two equivalently contributing species. The signal detected at $400.3 \mathrm{eV}$ (red curve) can be assigned to nitrogen atoms bound to three carbon atoms while the contribution at $399.0 \mathrm{eV}$ (blue curve) is characteristic for $\mathrm{sp}^{2}$-hybridized nitrogen atoms in the heterocyclic ring forming $\mathrm{C}$ $\mathrm{N}=\mathrm{C}$-bonds. In comparison, the fraction of $\mathrm{N}-\mathrm{C}_{3}$ is species is slightly reduced for $\mathrm{p}-\mathrm{C}_{3} \mathrm{~N}_{4}$. The C 1s region (Figure 5e) shows characteristic energy levels for carbon atoms bound to three nitrogen atoms in heterocycles at $288.8 \mathrm{eV}$. Only a minor component at $285.1 \mathrm{eV}$ caused by $\mathrm{C}-\mathrm{C}$ bonds, likely located at surface defects, can be detected. As the atomic ratio $\mathrm{C} / \mathrm{N}$ is calculated to of 0.73 for $\mathrm{g}-\mathrm{C}_{3} \mathrm{~N}_{4}$, we propose a structure close to the ideal heptazine-based polymer shown in Figure 5f. ${ }^{[61]}$ For p- $\mathrm{C}_{3} \mathrm{~N}_{4}$, the signal corresponding to carbon atoms in the triazine unit (blue curve in Figure $\mathbf{5 h}$ ) is slightly shifted to $288.6 \mathrm{eV}$ indicating a change in binding situation. As the atomic $\mathrm{C} / \mathrm{N}$ ratio is enhanced to 0.75 , we attribute this to the formation of a triazine-heptazine polymer as reported previously in literature. ${ }^{[44 c, 62]}$ The crystalline structure determined by powder X-ray diffraction (PXRD) measurements (Supporting Information Figure S14) showing a very broad reflex, corresponding to the interplane stacking expected for the synthesized materials. The observed shift of the (002)-reflex to higher interlayer distances for $\mathrm{C} @ \mathrm{~N}$ as compared to $\mathrm{g}-\mathrm{C}_{3} \mathrm{~N}_{4}$ and $\mathrm{p}-\mathrm{C}_{3} \mathrm{~N}_{4}$, consistent with the proposed structures and confirms the determined structures.

\subsection{Optical Properties and modification of graded porous materials.}

As a final distinction between the synthesized materials, we investigated their optical properties using solid-state UV-VIS spectroscopy (Figure 6(a)). As the application fields for doped carbons asks for high conductivity $\left.{ }^{[39}, 41 \mathrm{~b}, 63\right]$, while $\mathrm{g}-\mathrm{C}_{3} \mathrm{~N}_{4}$ is mainly discussed in photocatalysis $^{[46 \mathrm{~b}, 64]}$, the modification of the electronic structure is highly important for future 
applications. We herein show the first synthetic route capable of creating graded nanoporous materials exhibiting these highly adjustable properties. This is demonstrated by a comparison of the synthesized $\mathrm{N} @ \mathrm{C}, \mathrm{g}-\mathrm{C}_{3} \mathrm{~N}_{4}$ and $\mathrm{p}-\mathrm{C}_{3} \mathrm{~N}_{4}$ with different internal structures. In accordance with the determined structure of a $\mathrm{sp}^{2}$-hybridizied nitrogen doped carbon material, $\mathrm{N} @ \mathrm{C}$ (Supporting Information Figure S15) is characterized by a strong absorbance over the whole visible range. In contrast, $\mathrm{g}-\mathrm{C}_{3} \mathrm{~N}_{4}$ materials (blue curves) show an absorption in the visible range starting from $\sim 500 \mathrm{~nm}$. Importantly, the band gap of $\mathrm{g}-\mathrm{C}_{3} \mathrm{~N}_{4}$ can be modified by the usage of different precursor systems, as this results in slightly altered $\mathrm{C} / \mathrm{N}$ ratios and therefore a modified binding situation. ${ }^{[65]}$ This concept can be transferred to tailored gradient porous $\mathrm{C}_{3} \mathrm{~N}_{4}$ materials, as shown by the synthesis of $\mathrm{g}-\mathrm{C}_{3} \mathrm{~N}_{4}$ using pure cyanamide or cyanamide-guanidine mixtures (see Supporting Information Fig. S16) as precursors. The resulting gradient porous monoliths are characterized by shifted band gaps in the range $2.55 \mathrm{eV}$ and $2.77 \mathrm{eV}$ as calculated from Tauc plots (inset in Figure 6(a)).
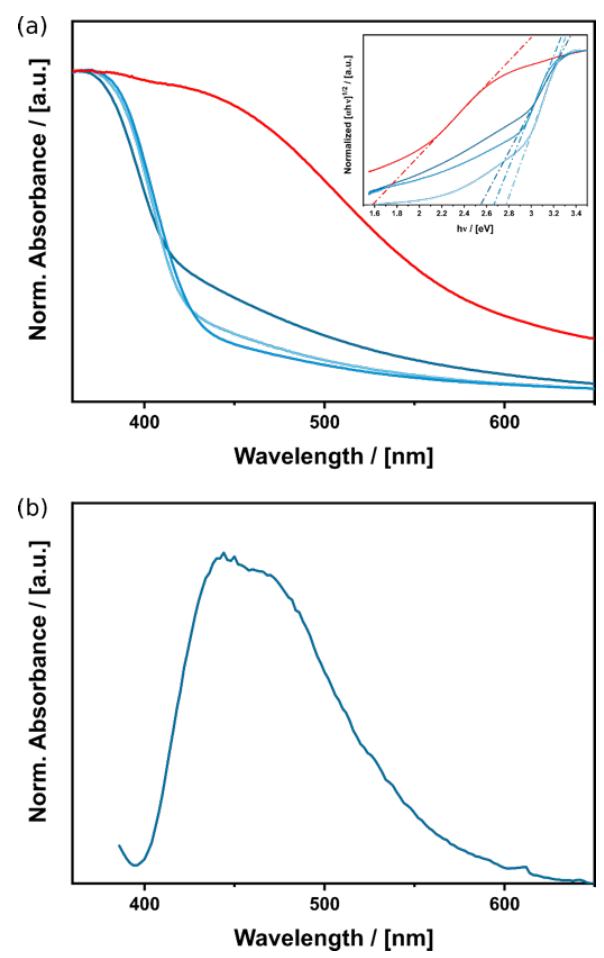

Figure 1: Solid state UV-VIS absorption measurements (a) normalized to the absorbance at $366 \mathrm{~nm}$ with Tauc plots as inset of the g-C3N4 samples (blue) synthesized using mixtures of cyanamide-urea mixture (dark blue), cyanamide-guanidine mixtures (blue) and pure cyanamide (light blue) and p-C3N4 (red) and a representative PL spectrum of g-C3N4. 
As matching photonic properties with the electronic band gap can lead to highly enhanced photoelectronic features $^{[7]}$, the independent adjustment of pore sizes and electronic properties is highly important. Therefore, the modified optical properties of $\mathrm{g}-\mathrm{C}_{3} \mathrm{~N}_{4}$ (red curve), shown by the broadened and red shifted absorbance in the solid state, are important. This results in a shift of the optical band gap to a comparable low value of $1.63 \mathrm{eV}$ (inset in Figure 6). This is a result of the desired triazine-heptazine based polymeric structure, realized by a deliberately chosen precursor approach, resulting in the formation of a donor-acceptor structure ${ }^{[44 a, 62]}$, showing the tailored variability of the optical properties in semiconducting materials with gradient porosity. For proof of concept, we have recorded photoluminescence (PL) spectra of a representative case (Figure 6(b)). As expected, the emission signal $\left(\lambda_{\max }=443 \mathrm{~nm}\right)$ is red shifted with respect to the adsorption edge.

\section{Conclusion}

Possible applications of gradient porous materials have been limited due to a lack of synthetic methods. We use preparative ultracentrifugation as a powerful tool to overcome this issue. By carefully analyzing the sedimentation parameters and a subsequent transfer towards PUC, SiO2-NPs can be fractionated in water or concentrated precursor-solutions. The formed colloidal glasses with gradually changing particles size along the direction of the centrifugal force can then be directly used as templating structures, which was not possible until now. We demonstrate this by developing a highly flexible synthetic method towards graded porous materials ranging from carbon over nitrogen doped carbon to $\mathrm{C}_{3} \mathrm{~N}_{4}$, which was not possible so far. In addition to the described synthesis methods, relying on aqueous systems, $\mathrm{SiO}_{2}-\mathrm{NPs}$ offer the advantage of a compatibility with various dispersion media, making a wide range of precursors applicable, as shown by the synthesis of $g-\mathrm{C}_{3} \mathrm{~N}_{4}$ and $\mathrm{p}-\mathrm{C}_{3} \mathrm{~N}_{4}$ with significantly altered properties. A further combination with our previous finding of the possibility of the 
introduction of chemical gradients into carbon materials by PUC ${ }^{[50-51]}$, will allow the synthesis of tailor made catalysts exhibiting graded porosity, significantly enhancing the field and possible applications of FGM.

Since local concentration and the control of mass and photon propagation is an important factor for catalytic processes ${ }^{[66]}$, we propose that the herein described materials offer an ideal platform to investigate possible synergistic effects in heterogeneous electro- and photocatalysis. Especially the investigations on the impact of a gradient in pore sizes on the optical properties and light propagation in our synthesized materials will allow an improvement of photocatalytic activity, for example by highly localized light trapping. ${ }^{[67]}$ Due to the high refractive index of $\mathrm{C}_{3} \mathrm{~N}_{4}$, a resonant light transport seems likely for pore sizes in the range of visible light ${ }^{[18 b]}$. In combination with prolonged retention times of photons inside of photonic glasses ${ }^{[18 a]}$ an enhancement in light harvesting is to be expected, if the photonic structure, resulting as a function of the pore sizes matches the energy levels of the electronic band gap. However, as our study is, to the best of our knowledge, the first report of a gradient porous semiconductors with pore sizes in the regime of the visible light, the necessary complex measurement setups will have to be developed first, which asks for further research.

\section{Experimental Section/Methods}

Materials. Tetraethylorthosilicate (TEOS, > $98 \%$ ), cyanamide ( >99\%), 5-aminotetrazole (5AT. $>97 \%)$ and dicyandiamide (>99\%) were purchased from Sigma-Aldrich. Guanidine carbonate ( $>98 \%$ ) and melamine ( $>99 \%$ ) were purchased at abcr. Resorcinol ( $>99 \%)$ and urea ( $>99.5 \%)$ were purchased from Merck. Aqueous formaldehyde solution (37 wt \%) was purchased from Roth. Guanidine hydrochloride ( $>98 \%$ ) was purchased from Alfa Aesar. Preparation of template particles. $\mathrm{SiO}_{2}-\mathrm{Nanoparticles}$ were synthesized by the Stoeber method. ${ }^{[53]} \mathrm{SiO}_{2}-129$ was synthesized by first dissolving concentrated aqueous $\mathrm{NH}_{4} \mathrm{OH}-$ 
solution $(4.5 \mathrm{~mL})$ in ethanol $(100 \mathrm{~mL})$. The solution was heated to $60^{\circ} \mathrm{C}$ under stirring. After $30 \mathrm{~min}$, TEOS $(4.5 \mathrm{~mL})$ was rapidly injected into the solution. The reaction was allowed to proceed for $24 \mathrm{~h} . \mathrm{SiO}_{2}-129$ was isolated by centrifugation and washed 2 times with Ethanol and subsequently 2 times with water. The obtained powder was dried and then dispersed and stored in water. The solid content was determined to be $11.8 \mathrm{wt} \%$. $\mathrm{SiO}_{2}-310$ was synthesized by heating a solution of MilliQ-grade water $(72 \mathrm{~mL})$, concentrated aqueous $\mathrm{NH}_{4} \mathrm{OH}$-solution $(15 \mathrm{~mL})$ and ethanol $(100 \mathrm{~mL}) 60^{\circ} \mathrm{C}$. The solution was heated to $30^{\circ} \mathrm{C}$ under stirring. After $30 \mathrm{~min}$, TEOS $(20 \mathrm{~mL})$ was rapidly injected into the solution. The reaction was allowed to proceed for $2 \mathrm{~h}$. $\mathrm{SiO}_{2}-310$ was isolated by centrifugation and washed 2 times with Ethanol and subsequently 2 times with water. The obtained powder was dried and then dispersed and stored in water. The solid content was determined to be $10.6 \mathrm{wt} \%$.

Preparation of $\mathrm{SiO}_{2}$-Assemblies. Dispersions of $\mathrm{SiO}_{2}-129$ and $\mathrm{SiO}_{2}-310$ were mixed in a volumetric ratio of 1:1 and diluted with MilliQ-grade water. The samples were then fractionated in thickwall polypropylene $(13 \times 51 \mathrm{~mm})$ centrifuge tubes by ultracentrifugation using a Beckman a Beckman Optima L-70 ultracentrifuge (Beckman instruments) equipped with a swinging-bucket rotor (SW 55 Ti, Beckman Instruments). For the detailed parameters (Volume ratios, centrifugal force), see the Supporting Info. After centrifugation, the supernatant was carefully removed, and the samples were dried at ambient conditions.

Preparation of gradient porous materials by melt infiltration. $\mathrm{SiO}_{2}$ assemblies formed after PUC were overlayed with excess $5 \mathrm{AT}$ in a corundum crucible. The sample was heated to $550{ }^{\circ} \mathrm{C}$ over $12 \mathrm{~h}$. During heat up, the monoliths were infiltrated. The full conversion to $g-\mathrm{C}_{3} \mathrm{~N}_{4}$ was achieved after holding the temperature at $550{ }^{\circ} \mathrm{C}$ for $2 \mathrm{~h}$. After cooling down, $\mathrm{SiO}_{2}$ was removed from the monolithic composites by etching in aqueous $20 \mathrm{wt} \%$ HF-solutions. After washing of the material with water, monolithic porous materials were obtained after drying at $90{ }^{\circ} \mathrm{C}$ under ambient pressure. 
Preparation of gradient porous materials by in-situ infiltration. For materials synthesis, aqueous dispersions of $\mathrm{SiO}_{2}-129$ and $\mathrm{SiO}_{2}-310$ (volumetric ratio of 1:1) were mixed with the respective concentrated precursor solutions (see the Supporting Info detailed parameters). The samples were infiltrated during fractionation in thickwall polypropylene $(13 \mathrm{~mm} \times 51 \mathrm{~mm})$ centrifuge tubes by ultracentrifugation using a Beckman a Beckman Optima L-70 ultracentrifuge (Beckman instruments) equipped with a swinging-bucket rotor (SW $55 \mathrm{Ti}$, Beckman Instruments). After centrifugation, the supernatant was carefully removed and the samples were dried at $90{ }^{\circ} \mathrm{C}$ for 3 days. The resulting monolithic, infiltrated samples were then pyrolyzed in a nitrogen atmosphere at $550{ }^{\circ} \mathrm{C}$ for $2 \mathrm{~h}$ with a heating ramp over $12 \mathrm{~h}$. After cooling down, $\mathrm{SiO}_{2}$ was removed from the monolithic composites by etching in aqueous $20 \mathrm{wt} \%$ HF-solutions. After washing of the material with water, monolithic porous materials were obtained after drying at $90{ }^{\circ} \mathrm{C}$ under ambient pressure.

Materials Characterization. For SEM imaging, monolithic carbon samples were cut into two pieces along the direction of the centrifugal force. Images were then acquired at several positions along the resulting cross-section using a Zeiss Gemini SEM 500 operating at $3-15 \mathrm{kV}$. EDX data were recorded using an Oxford Instruments Ultim ${ }^{\circledR}$ Max with a $100 \mathrm{~mm}^{2}$ Silicon Drift Detector mounted onto the microscope. X-ray diffraction analysis was performed with a Bruker AXS D8 Advance diffractometer using $\mathrm{Cu}-\mathrm{K} \alpha$ radiation. TGA-measurements were performed using a Netzsch STA 429 F3 Jupiter. XPS measurements were performed by Tascon GmbH, Sulzbach, Germany using a PHI Quantera SXM with Al- Ka radiation. FT-IR spectra were obtained with a Perkin-Elmer Spectrum 100 with ATR measurement unit. $\mathrm{N}_{2}-$ physisorption measurements were conducted on a Micromeritics TriStar 3000. UV VIS measurements were recorded using a Cary-5000 including integrated sphere, Agilent 8453. AUC measurements were conducted using an Optima XL-I analytical ultracentrifuge (Beckman Coulter, Palo Alto, CA, USA) with Rayleigh interference optics in a titanium measurement cell (Nanolytics, Potsdam, Germany). The optical pathway length of the cell was $12 \mathrm{~mm}$. 


\section{Supporting Information}

Supporting Information is available from the Web or from the author.

\section{Acknowledgements}

We thank the Carl-Zeiss foundation for funding Jochen Bahner by a full Ph. D.scholarship. We gratefully acknowledge technical/instrumental support from the German Research Foundation (DFG) via SFB1214 Project Z1 Particle Analysis Center. We acknowledge Rose Rosenberg for assisting in AUC measurements. The authors thank Nele Klinkenberg, Ulrich Bahnmüller and Sebastian Sutter for their valuable feedback from proofreading the manuscript.

\section{References}

[1] a) M. Hartmann and W. Schwieger, Chem Soc Rev 2016, 45, 3311-3312; b) Y. Li, Z.-Y.

Fu and B.-L. Su, Advanced Functional Materials 2012, 22, 4634-4667.

[2] M. H. Sun, S. Z. Huang, L. H. Chen, Y. Li, X. Y. Yang, Z. Y. Yuan and B. L. Su, Chem Soc Rev 2016, 45, 3479-3563.

[3] C. M. Parlett, K. Wilson and A. F. Lee, Chem Soc Rev 2013, 42, 3876-3893.

[4] a) S. Polarz and M. Antonietti, Chemical Communications 2002, 2593-2604; b) O. D.

Velev and E. W. Kaler, Advanced Materials 2000, 12, 531-534.

[5] E. Yablonovitch, T. J. Gmitter and K. M. Leung, Physical Review Letters 1991, 67, 2295 2298.

[6] J. C. Knight, Nature 2003, 424, 847-851.

[7] E. Armstrong and C. O'Dwyer, Journal of Materials Chemistry C 2015, 3, 6109-6143.

[8] F. Temerov, B. Ankudze and J. J. Saarinen, Materials Chemistry and Physics 2020, 242, 122471.

[9] L. Zheng, H. Cheng, F. Liang, S. Shu, C. K. Tsang, H. Li, S.-T. Lee and Y. Y. Li, Journal of Physical Chemistry C 2012, 116, 5509-5515.

[10] J. I. L. Chen and G. A. Ozin, Journal of Materials Chemistry 2009, 19, 2675-2678.

[11] X. Zhang and S. John, Journal of Materials Chemistry A 2020, 8, 18974-18986. 
[12] a) J. Liu, H. Zhao, M. Wu, B. Van der Schueren, Y. Li, O. Deparis, J. Ye, G. A. Ozin, T. Hasan and B.-L. Su, Advanced Materials 2017, 29, 1605349; b) S. Y. Lim, C. S. Law, M.

Markovic, J. K. Kirby, A. D. Abell and A. Santos, ACS Applied Materials \& Interfaces 2018, $10,24124-24136$.

[13] J. Low, L. Zhang, B. Zhu, Z. Liu and J. Yu, ACS Sustainable Chemistry \& Engineering 2018, 6, 15653-15661.

[14] P. D. Garcia, R. Sapienza and C. Lopez, Adv Mater 2010, 22, 12-19.

[15] M. Gaio, M. Peruzzo and R. Sapienza, Optics Letters 2015, 40, 1611-1614.

[16] L. Schertel, L. Siedentop, J.-M. Meijer, P. Keim, C. M. Aegerter, G. J. Aubry and G. Maret, Advanced Optical Materials 2019, 7, 1900442.

[17] P. W. Anderson, Philosophical Magazine B 1985, 52, 505-509.

[18] a) M. Chen, D. Fischli, L. Schertel, G. J. Aubry, B. Häusele, S. Polarz, G. Maret and H. Cölfen, Small 2017, 13, 1701392; b) L. Schertel, I. Wimmer, P. Besirske, C. M. Aegerter, G. Maret, S. Polarz and G. J. Aubry, Physical Review Materials 2019, 3, 015203.

[19] a) J. J. do Rosário, P. N. Dyachenko, R. Kubrin, R. M. Pasquarelli, A. Y. Petrov, M. Eich and G. A. Schneider, ACS Applied Materials \& Interfaces 2014, 6, 12335-12345; b) S.-H. Kim, S. Magkiriadou, D. K. Rhee, D. S. Lee, P. J. Yoo, V. N. Manoharan and G.-R. Yi, ACS Applied Materials \& Interfaces 2017, 9, 24155-24160.

[20] G. H. Lee, J. Y. Sim and S.-H. Kim, ACS Applied Materials \& Interfaces 2016, 8, 12473-12480.

[21] Y. Häntsch, G. Shang, A. Petrov, M. Eich and G. A. Schneider, Advanced Optical Materials 2019, 7, 1900428.

[22] B. H. Rabin and I. Shiota, MRS Bulletin 1995, 20, 14-18.

[23] J. Genzer, Annual Review of Materials Research 2012, 42, 435-468.

[24] a) W. Du and C. Gao, Macromol Biosci 2019, 19, e1900292; b) Z. Wang, ACS Nano 2018, $12,1273-1284$. 
[25] M. M. Collinson and D. A. Higgins, Langmuir 2017, 33, 13719-13732.

[26] J. Werner, B. Linner-Krčmar, W. Friess and P. Greil, Biomaterials 2002, 23, 4285-4294.

[27] P. Fratzl and R. Weinkamer, Progress in Materials Science 2007, 52, 1263-1334.

[28] X. Miao and D. Sun, Materials 2009, 3, 26-47.

[29] T. Yamaoka, Analytica Chimica Acta 1998, 372, 91-98.

[30] a) J. E. Trachtenberg, J. K. Placone, B. T. Smith, J. P. Fisher and A. G. Mikos, J Biomater Sci Polym Ed 2017, 28, 532-554; b) S. Sultan and A. P. Mathew, Nanoscale 2018, $10,4421-4431$.

[31] a) A. Ahmed, J. Smith and H. Zhang, Chemical Communications 2011, 47, 11754-

11756; b) C. Stubenrauch, A. Menner, A. Bismarck and W. Drenckhan, Angewandte Chemie International Edition 2018, 57, 10024-10032.

[32] a) P. Gannon, S. Sofie, M. Deibert, R. Smith and V. Gorokhovsky, Journal of Applied Electrochemistry 2008, 39, 497-502; b) A. Macchetta, I. G. Turner and C. R. Bowen, Acta Biomaterialia 2009, 5, 1319-1327.

[33] M. Costantini, J. Jaroszewicz, Ł. Kozoń, K. Szlązak, W. Święszkowski, P. Garstecki, C. Stubenrauch, A. Barbetta and J. Guzowski, Angewandte Chemie International Edition 2019, $58,7620-7625$.

[34] D. Tan, Q. Li, B. Yang, X. Wang, S. Hu, Z. Wang, Y. Lei and L. Xue, Langmuir 2019, $35,5864-5870$.

[35] a) A. Spinnrock and H. Colfen, Chemistry- A European Journal 2019, 25, 10026-10032;

b) X. Xu and H. Colfen, Nanomaterials 2021, 11 .

[36] a) X. Xu and H. Colfen, ChemPhysChem 2019, 20, 1799-1803; b) X. Xu, T. Franke, K.

Schilling, N. Sommerdijk and H. Colfen, Nano Lett 2019, 19, 1136-1142.

[37] M. Chen, H. Cölfen and S. Polarz, ACS Nano 2015, 9, 6944-6950.

[38] M. Chen, K. Hagedorn, H. Colfen and S. Polarz, Adv Mater 2017, 29.

[39] J. P. Paraknowitsch and A. Thomas, Energy \& Environmental Science 2013, 6. 
[40] W. Shen and W. Fan, J. Mater. Chem. A 2013, 1, 999-1013.

[41] a) E. Luo, M. Xiao, J. Ge, C. Liu and W. Xing, J. Mater. Chem. A 2017, 5, 21709-21714;

b) D. Guo, H. Wei, X. Chen, M. Liu, F. Ding, Z. Yang, Y. Yang, S. Wang, K. Yang and S. Huang, Journal of Materials Chemistry A 2017, 5, 18193-18206; c) Z. Yi, Z. Zhang, S. Wang and G. Shi, Journal of Materials Chemistry A 2017, 5, 519-523.

[42] H. Wang, J. Jia, P. Song, Q. Wang, D. Li, S. Min, C. Qian, L. Wang, Y. F. Li, C. Ma, T. Wu, J. Yuan, M. Antonietti and G. A. Ozin, Angew Chem Int Ed Engl 2017, 56, 7847-7852.

[43] K. Ogata, J. F. D. Chubaci and F. Fujimoto, Journal of Applied Physics 1994, 76, 37913796.

[44] a) D. Dontsova, S. Pronkin, M. Wehle, Z. Chen, C. Fettkenhauer, G. Clavel and M. Antonietti, Chemistry of Materials 2015, 27, 5170-5179; b) A. Savateev, S. Pronkin, J. D. Epping, M. G. Willinger, C. Wolff, D. Neher, M. Antonietti and D. Dontsova, ChemCatChem 2017, 9, 167-174; c) G. Zhang, M. Liu, T. Heil, S. Zafeiratos, A. Savateev, M. Antonietti and X. Wang, Angewandte Chemie International Edition 2019, 58, 14950-14954.

[45] Y. Wang, X. Wang and M. Antonietti, Angewandte Chemie International Edition 2012, $51,68-89$.

[46] a) A. Kumar, P. Raizada, P. Singh, R. V. Saini, A. K. Saini and A. HosseiniBandegharaei, Chemical Engineering Journal 2020, 391, 123496; b) K. R. Reddy, C. H. V. Reddy, M. N. Nadagouda, N. P. Shetti, S. Jaesool and T. M. Aminabhavi, Journal of Environmental Management 2019, 238, 25-40; c) J. Oh, J. M. Lee, Y. Yoo, J. Kim, S.-J. Hwang and S. Park, Applied Catalysis B: Environmental 2017, 218, 349-358; d) A. Mishra, A. Mehta, S. Basu, N. P. Shetti, K. R. Reddy and T. M. Aminabhavi, Carbon 2019, 149, 693721; e) P. Lianos, Applied Catalysis B: Environmental 2017, 210, 235-254; f) W. Zhan, L. Sun and X. Han, Nano-Micro Letters 2019, 11; g) Y. Shi, J. Huang, G. Zeng, W. Cheng and J. Hu, Journal of Membrane Science 2019, 584, 364-392; h) J. H. Pan, H. Dou, Z. Xiong, C. Xu, J. Ma and X. S. Zhao, Journal of Materials Chemistry 2010, 20. 
[47] a) Y. Li, X. Li, H. Zhang and Q. Xiang, Nanoscale Horiz 2020, 5, 765-786; b) S. N. Talapaneni, G. Singh, I. Y. Kim, K. AlBahily, A. H. Al-Muhtaseb, A. S. Karakoti, E. Tavakkoli and A. Vinu, Adv Mater 2020, 32, e1904635.

[48] Q. Liang, Z. Li, X. Yu, Z.-H. Huang, F. Kang and Q.-H. Yang, Advanced Materials $\mathbf{2 0 1 5}, 27,4634-4639$.

[49] L. Sun, M. Yang, J. Huang, D. Yu, W. Hong and X. Chen, Advanced Functional Materials 2016, 26, 4943-4950.

[50] J. Bahner, N. Klinkenberg, M. Frisch, L. Brauchle and S. Polarz, Advanced Functional Materials 2019, 29, 1904058.

[51] J. Bahner, N. Hug and S. Polarz, C 2021, 7, 22.

[52] a) X. Fan, Z. Xing, Z. Shu, L. Zhang, L. Wang and J. Shi, RSC Advances 2015, 5, 83238328; b) K. Muirhead, S. Earnshaw, C. J. Easton and A. Philbrook, Journal of Applied Polymer Science 2012, 125, E372-E377.

[53] W. Stöber, A. Fink and E. Bohn, Journal of Colloid and Interface Science 1968, 26, 6269.

[54] O. D. Velev and S. Gupta, Advanced Materials 2009, 21, 1897-1905.

[55] R. Piazza, S. Buzzaccaro, E. Secchi and A. Parola, Phys Biol 2013, 10, 045005.

[56] I. Arganda-Carreras, V. Kaynig, C. Rueden, K. W. Eliceiri, J. Schindelin, A. Cardona and H. Sebastian Seung, Bioinformatics 2017, 33, 2424-2426.

[57] C. T. Rueden, J. Schindelin, M. C. Hiner, B. E. DeZonia, A. E. Walter, E. T. Arena and K. W. Eliceiri, BMC Bioinformatics 2017, 18, 529.

[58] K. Sakaushi and M. Antonietti, Bulletin of the Chemical Society of Japan 2015, 88, 386398.

[59] S. Zhang, S. Tsuzuki, K. Ueno, K. Dokko and M. Watanabe, Angew Chem Int Ed Engl 2015, 54, 1302-1306. 
[60] M. Ayiania, M. Smith, A. J. R. Hensley, L. Scudiero, J.-S. McEwen and M. GarciaPerez, Carbon 2020, 162, 528-544.

[61] N. Hellgren, R. T. Haasch, S. Schmidt, L. Hultman and I. Petrov, Carbon 2016, 108, 242-252.

[62] G. Zhang, L. Lin, G. Li, Y. Zhang, A. Savateev, S. Zafeiratos, X. Wang and M. Antonietti, Angew Chem Int Ed Engl 2018, 57, 9372-9376.

[63] S. Dutta, A. Bhaumik and K. C. W. Wu, Energy Environ. Sci. 2014, 7, 3574-3592.

[64] L. Jiang, X. Yuan, Y. Pan, J. Liang, G. Zeng, Z. Wu and H. Wang, Applied Catalysis B: Environmental 2017, 217, 388-406.

[65] Z. Yang, K. Hu, X. Meng, Q. Tao, J. Dong, B. Liu, Q. Lu, H. Zhang, B. Sundqvist, P. Zhu, M. Yao and B. b. Liu, Carbon 2018, 130, 170-177.

[66] W. Wan, R. Zhang, M. Ma and Y. Zhou, Journal of Materials Chemistry A 2018, 6, 754775.

[67] X. Sheng, J. Liu, I. Kozinsky, A. M. Agarwal, J. Michel and L. C. Kimerling, Advanced Materials 2011, 23, 843-847. 